<smiles>CC(=O)c1c(NC(C)(C)C)oc(-c2c(N3CCSCC3)c3ccccc3oc2=O)c1C(C)=O</smiles><smiles>CCOC(C)=O</smiles>

\section{Crystal structure of 3,4-dimethyl 2-(tert-} butylamino)-5-[2-oxo-4-(thiomorpholin4-yl)-2H-chromen-3-yl]furan-3,4-dicarboxylate ethyl acetate hemisolvate

\author{
Tetsuji Moriguchi, ${ }^{a *}$ Venkataprasad Jalli, ${ }^{a}$ Suvratha \\ Krishnamurthy, ${ }^{a}$ Akihiko Tsuge ${ }^{a}$ and Kenji Yoza ${ }^{b}$ \\ a Department of Applied Chemistry, Graduate School of Engineering, Kyushu Institute \\ of Technology, 1-1 Sensui-cho, Tobata-ku, Kitakyushu 804-8550, Japan, and bapan \\ Bruker AXS K.K.3-9, Moriya-cho Kanagawaku Yokohama 221-0022, Japan. \\ *Correspondence e-mail: moriguch@che.kyutech.ac.jp
}

Received 26 October 2015; accepted 18 November 2015

Edited by W. T. A. Harrison, University of Aberdeen, Scotland

In the title hemisolvate, $\mathrm{C}_{25} \mathrm{H}_{28} \mathrm{~N}_{2} \mathrm{O}_{7} \mathrm{~S} \cdot 0.5 \mathrm{C}_{4} \mathrm{H}_{8} \mathrm{O}_{2}$, the thiomorpholine ring adopts a chair conformation, with the exocyclic $\mathrm{N}-\mathrm{C}$ bond in an equatorial orientation. The dihedral angle between the coumarin ring system (r.m.s. deviation $=0.044 \AA$ ) and the furan ring is $64.84(6)^{\circ}$. An intramolecular $\mathrm{N}-\mathrm{H} \cdots \mathrm{O}$ hydrogen bond closes an $S(6)$ ring. The ethyl acetate solvent molecule is disordered about a crystallographic inversion centre. In the crystal, the components are linked by $\mathrm{C}-\mathrm{H} \cdot \mathrm{O}$ and $\mathrm{C}-\mathrm{H} \cdots \mathrm{S}$ hydrogen bonds, generating a three-dimensional network.

Keywords: crystal structure; coumarins; thiomorpholine ring; hydrogen bonding.

CCDC reference: 1432824

\section{Related literature}

For the syntheses and properties of coumarins, see: Arango et al. (2010); Chodankar \& Seshadri (1985); Khan \& Kulkarni (1999); Kitamura et al. (2005); Luo et al. (2012); Sawa et al. (2006); Schiedel et al. (2001); Udaya Kumari et al. (2000); Zen et al. (2014).

\section{Experimental}

\subsection{Crystal data}

$\mathrm{C}_{25} \mathrm{H}_{28} \mathrm{~N}_{2} \mathrm{O}_{7} \mathrm{~S} \cdot 0.5 \mathrm{C}_{4} \mathrm{H}_{8} \mathrm{O}_{2}$ $M_{r}=544.61$

Monoclinic, $P 2_{1} / c$

$a=14.3733(17) \AA$

$b=16.1159$ (19) $\AA$

$c=11.7019(14) \AA$

$\beta=95.007(1)^{\circ}$

$V=2700.3(6) \AA^{3}$

$Z=4$

Mo $K \alpha$ radiation

$\mu=0.17 \mathrm{~mm}^{-1}$

$T=90 \mathrm{~K}$

$0.50 \times 0.40 \times 0.25 \mathrm{~mm}$

\subsection{Data collection}

Bruker APEXII diffractometer Absorption correction: multi-scan (SADABS; Bruker, 2009)

$T_{\text {min }}=0.853, T_{\max }=0.958$

25410 measured reflections 4757 independent reflections 4316 reflections with $I>2 \sigma(I)$ $R_{\text {int }}=0.022$

\subsection{Refinement}

$R\left[F^{2}>2 \sigma\left(F^{2}\right)\right]=0.032$

$w R\left(F^{2}\right)=0.086$

$S=1.02$

4757 reflections

375 parameters

\section{0 restraints}

$\mathrm{H}$-atom parameters constrained

$\Delta \rho_{\max }=0.26 \mathrm{e} \AA^{-3}$

$\Delta \rho_{\min }=-0.23 \mathrm{e}^{-3}$

Table 1

Hydrogen-bond geometry $\left(\AA{ }^{\circ}\right)$.

\begin{tabular}{lllll}
\hline$D-\mathrm{H} \cdots A$ & $D-\mathrm{H}$ & $\mathrm{H} \cdots A$ & $D \cdots A$ & $D-\mathrm{H} \cdots A$ \\
\hline $\mathrm{N} 2-\mathrm{H} 13 \cdots \mathrm{O} 4$ & 0.86 & 2.25 & $2.8255(17)$ & 125 \\
$\mathrm{C} 3-\mathrm{H} 2 \cdots$ O6 $^{\mathrm{i}}$ & 0.93 & 2.41 & $3.325(2)$ & 167 \\
$\mathrm{C} 12-\mathrm{H} 9 \cdots \mathrm{O}^{\mathrm{ii}}$ & 0.97 & 2.44 & $3.1531(18)$ & 130 \\
$\mathrm{C} 12-\mathrm{H} 10 \cdots \mathrm{O} 1 S^{\mathrm{iii}}$ & 0.97 & 2.48 & $3.208(5)$ & 132 \\
$\mathrm{C} 19-\mathrm{H} 15 \cdots \mathrm{O} 3$ & 0.96 & 2.42 & $3.0090(19)$ & 119 \\
$\mathrm{C} 23-\mathrm{H} 23 \cdots \mathrm{S} 1^{\text {iv }}$ & 0.96 & 2.78 & $3.5639(17)$ & 139 \\
$\mathrm{C} 25-\mathrm{H} 26 \cdots \mathrm{S} 1^{\mathrm{v}}$ & 0.96 & 2.83 & $3.7406(18)$ & 159 \\
\hline Symmetry codes: (i) $-x+2,-y,-z+1 ;($ ii) $-x+1,-y,-z ;$ (iii) $x, y, z-1 ;$ (iv) \\
$-x+1, y+\frac{1}{2},-z+\frac{1}{2} ;(\mathrm{v}) x,-y-\frac{1}{2}, z+\frac{1}{2}$.
\end{tabular}

Data collection: APEX2 (Bruker, 2009); cell refinement: SAINT (Bruker, 2009); data reduction: $S A I N T$; $\operatorname{program}(\mathrm{s})$ used to solve structure: SHELXS97 (Sheldrick, 2008); program(s) used to refine structure: SHELXL97 (Sheldrick, 2008); molecular graphics: Mercury (Macrae et al., 2008); software used to prepare material for publication: SHELXL97.

\section{Acknowledgements}

We are grateful to the Center for Instrumental Analysis, Kyushu Institute of Technology (KITCIA) for the X-ray analysis. This research was supported financially by JSPS KAKENH grant No. 15 K05611. 
Supporting information for this paper is available from the $\mathrm{IUCr}$ electronic archives (Reference: HB7531).

\section{References}

Arango, V., Robledo, S., Séon-Méniel, B., Figadère, B., Cardona, W., Sáez, J. \& Otálvaro, F. (2010). J. Nat. Prod. 73, 1012-1014.

Bruker (2009). APEX2, SAINT and SADABS. Bruker AXS Inc., Madison, Wisconsin, USA.

Chodankar, N. K. (1985). Dyes Pigm. 6, 331-340.

Khan, I. A. \& Kulkarni, M. V. (1999). Indian. J. Chem. Sect. B, 38, 491-494.

Kitamura, N., Kohtani, S. \& Nakagaki, R. (2005). J. Photochem. Photobiol. Photochem. Rev. 6, 168-185.
Luo, X., He, W., Yin, H., Li, Q., Liu, Q., Huang, Y. \& Zhang, S. (2012). Molecules, 17, 6944-6952.

Macrae, C. F., Bruno, I. J., Chisholm, J. A., Edgington, P. R., McCabe, P., Pidcock, E., Rodriguez-Monge, L., Taylor, R., van de Streek, J. \& Wood, P. A. (2008). J. Appl. Cryst. 41, 466-470.

Sawa, M., Hsu, T. L., Itoh, T., Sugiyama, M., Hanson, S. R., Vogt, P. K. \& Wong, C. H. (2006). Proc. Natl Acad. Sci. USA, 103, 12371-12376.

Schiedel, M. S., Briehn, C. A. \& Bäuerle, P. (2001). Angew. Chem. Int. Ed. 40, 4677-4680.

Sheldrick, G. M. (2008). Acta Cryst. A64, 112-122.

Udaya Kumari, T., David Krupadanam, G. L. \& Srimannarayana, G. (2000). Indian. J. Chem. Sect. B, 39, 62-64.

Zen, A. Z., Aylott, J. W. \& Chan, W. C. (2014). Tetrahedron Lett. 55, $5521-$ 5524. 


\section{supporting information}

Acta Cryst. (2015). E71, o1003-o1004 [https://doi.org/10.1107/S2056989015021970]

Crystal structure of 3,4-dimethyl 2-(tert-butylamino)-5-[2-oxo-4-(thiomorpholin-4-yl)-2H-chromen-3-yl]furan-3,4-dicarboxylate ethyl acetate hemisolvate

\section{Tetsuji Moriguchi, Venkataprasad Jalli, Suvratha Krishnamurthy, Akihiko Tsuge and Kenji Yoza}

\section{S1. Structural commentary}

Coumarins analogs having furan heterocycle have gained significant importance because of their properties as antileishmania panamensis, dyes and fluorescent sensors. For the activity related reports of furyl coumarins, see: Arango et al. (2010); Zen et al. (2014); Schiedel et al. (2001); Kitamura et al. (2005). Natural furyl coumarin derivatives extracted from plants such as microminutin, micromelin, psoralen, 8-methoxypsoralen have important properties in medicinal chemistry and bio photochemistry. For the activity related reports of natural furyl coumarins, see: Luo et al. (2012). It was well documented that by introducing a heteroaromatic substituent at 3-position the absorption and emission maxima of coumarin scaffold can be improved because of extended $\pi$ conjugation and consequently their optoelectronic properties can be improved. Due to their versatile properties a variety of 3-heteroaryl coumarin derivatives have been synthesized and tested for their optoelectronic properties. For the optoelectronic properties of coumarin derivatives, see: Sawa et al. (2006). For the synthesis related reports of 3-furyl coumarin derivatives, see: Chodankar et al. (1985); Khan \& Kulkarni (1999); Udaya Kumari et al. (2000). Thus, the elucidation of the crystal structures of coumarin derivatives has attracted much attention. Here,we report the crystal structure of the title compound, (I).

\section{S2. Synthesis and crystallization,}

A solution of 4-thiomorpholino-3-formyl coumarin (1 mmol), dmethyl acetylenedicarboxylate (1 mmol), t-butyl isocyanide $(1 \mathrm{mmol})$ were refluxed at $80^{\circ} \mathrm{C}$ for $3 \mathrm{~h}$. The volatiles were removed under reduced pressure. The crude reaction mixture was subjected to column chromatography using EtOAc/Hexane mobile phase. The title compound was isolated as yellow color solid with $80 \%$ yield. Yellow prisms were obtained by vapour diffusion method at room temperature, i.e., hexane vapour was allowed to diffuse into an EtOAc solution of 4-thiomorpholino-3-(2-N-t-butylamino-3,4-dimethylcarboxylate-5-furyl) 2H-1-benzopyran-2-one at room temperature.

mp 107-109 ${ }^{\circ} \mathrm{C}$; IR; $v_{\max }(\mathrm{KBr}) 3288,1732,1728,1667,1658,1618,1418,1240,1041 \mathrm{~cm}^{-1} ; \delta_{\mathrm{H}}\left(500 \mathrm{MHz} \mathrm{CDCl}_{3}\right) 7.69$ $(1 \mathrm{H}, \mathrm{d}), 7.52(1 \mathrm{H}, \mathrm{t}), 7.28-7.33(2 \mathrm{H}, \mathrm{dd}), 7.05(1 \mathrm{H}, \mathrm{s}), 3.78(3 \mathrm{H}, \mathrm{s}), 3.75(3 \mathrm{H}, \mathrm{s}), 3.39-3.48(4 \mathrm{H}, \mathrm{m}), 2.77-2.81(4 \mathrm{H}$, m), $1.44(9 \mathrm{H}, \mathrm{s}) ; \delta_{\mathrm{C}}\left(125 \mathrm{MHz}, \mathrm{CDCl}_{3}\right)$ 165.6, 163.9, 163.0, 161.2, 160.6, 153.4, 138.2, 132.4, 125.1, 123.7, 118.7, 118.1, 117.7, 103.6, 87.8, 53.2, 52.7, 51.8, 51.3, 28.0; LCMS: $\mathrm{MH}^{+}, 501$. 


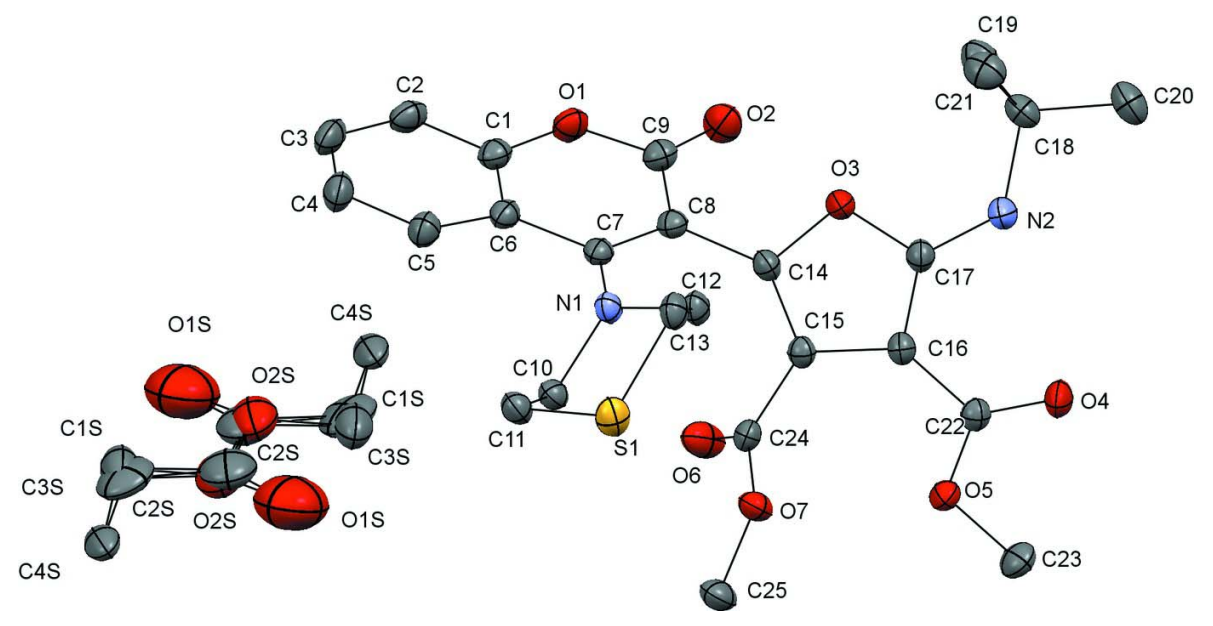

Figure 1

Molecular configuration and atom-numbering scheme for the title compound with displacement ellipsoids drawn at the $50 \%$ probability level. Hydrogen atoms are omitted for clarity.

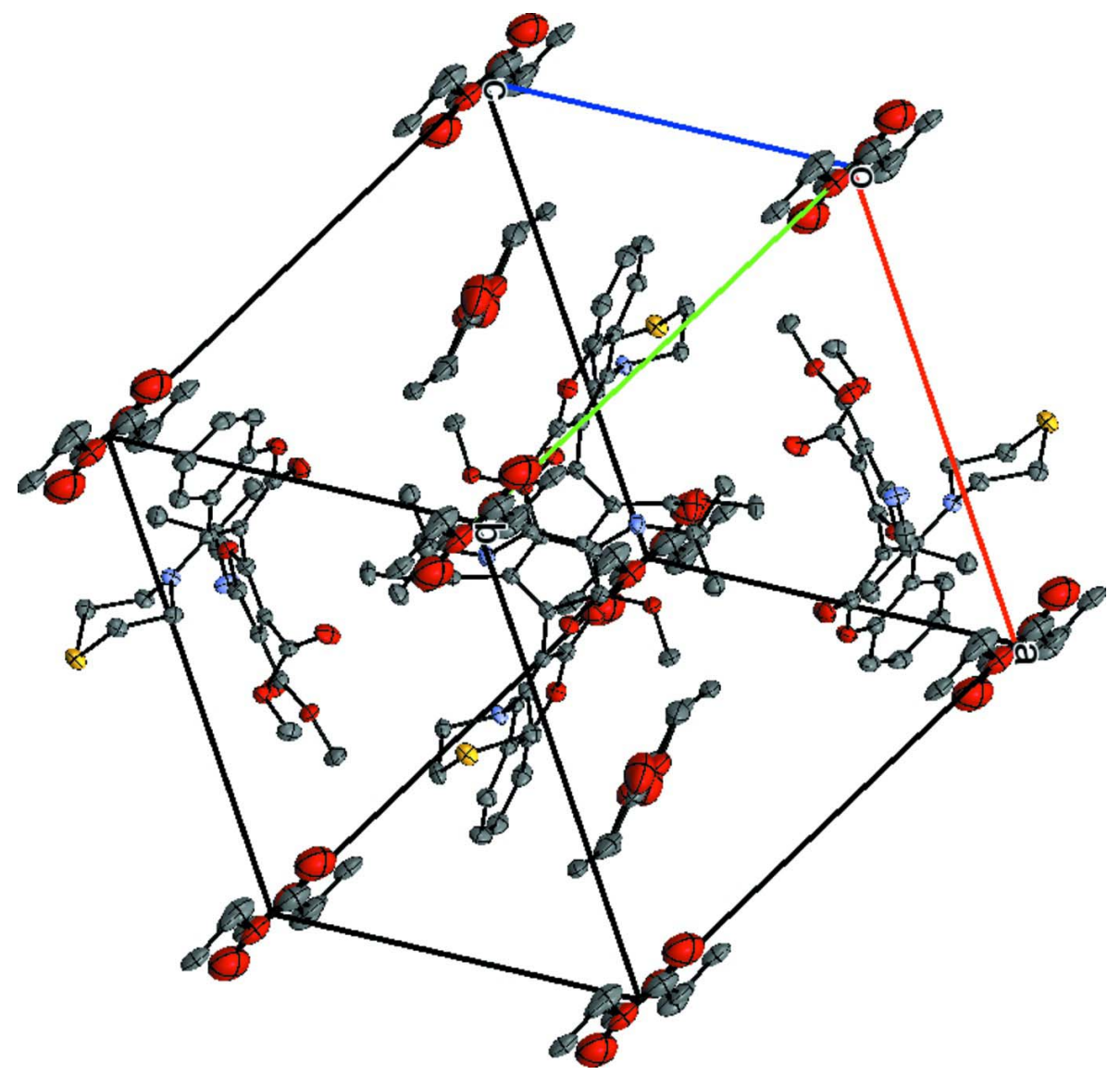

Figure 2

Crystal packing diagram of the title compound. 


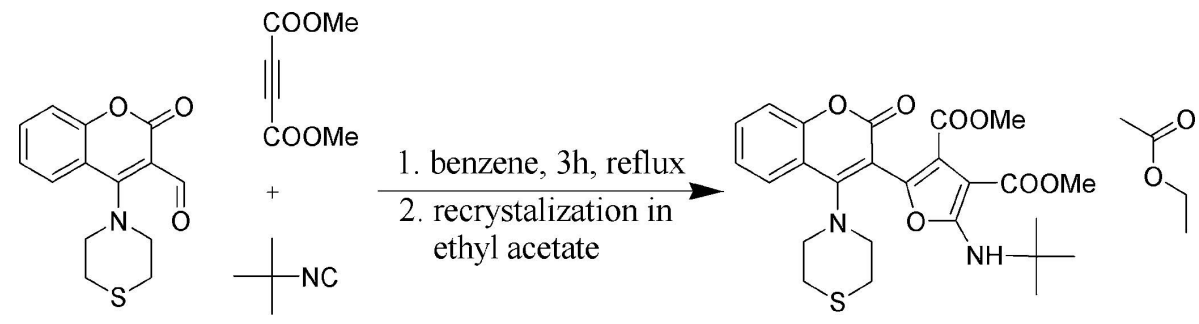

Figure 3

Chemical scheme of title compound with solvent molecule. In the cystal system the main molecule and solvent molecule was found in 1:0.5 ratio.

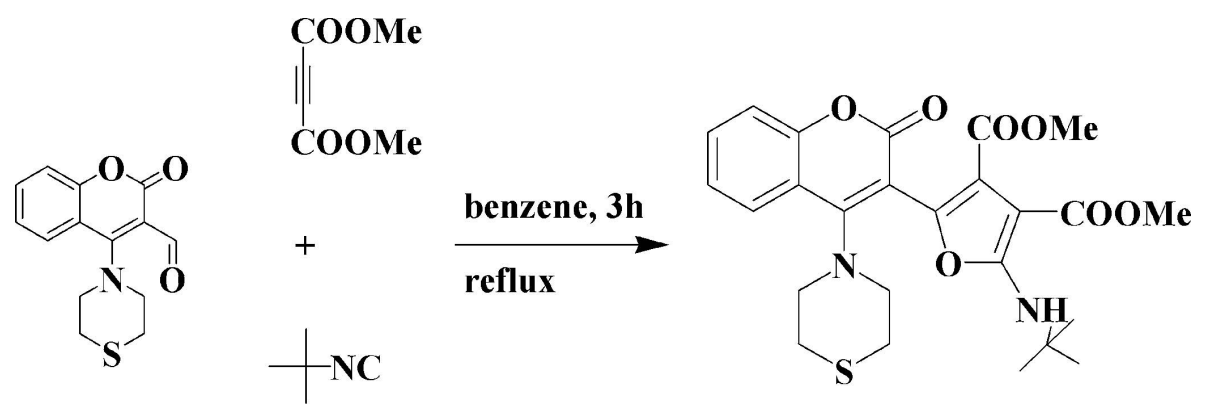

Figure 4

Synthesis of title compound (I).

3,4-Dimethyl 2-(tert-butylamino)-5-[2-oxo-4-(thiomorpholin-4-yl)-2H-chromen-3-yl]furan-3,4-dicarboxylate ethyl acetate hemisolvate

Crystal data

$\mathrm{C}_{25} \mathrm{H}_{28} \mathrm{~N}_{2} \mathrm{O}_{7} \mathrm{~S} \cdot 0.5 \mathrm{C}_{4} \mathrm{H}_{8} \mathrm{O}_{2}$

$M_{r}=544.61$

Monoclinic, $P 2_{1} / c$

Hall symbol: -P 2ybc

$a=14.3733$ (17) $\AA$

$b=16.1159$ (19) $\AA$

$c=11.7019(14) \AA$

$\beta=95.007(1)^{\circ}$

$V=2700.3(6) \AA^{3}$

$Z=4$

$F(000)=1152$

$D_{\mathrm{x}}=1.340 \mathrm{Mg} \mathrm{m}^{-3}$

Mo $K \alpha$ radiation, $\lambda=0.71073 \AA$

Cell parameters from 25410 reflections

$\theta=1.4-25.0^{\circ}$

$\mu=0.17 \mathrm{~mm}^{-1}$

$T=90 \mathrm{~K}$

Prism, yellow

$0.50 \times 0.40 \times 0.25 \mathrm{~mm}$

\section{Data collection}

\section{Bruker APEXII}

diffractometer

Radiation source: fine-focus sealed tube

Graphite monochromator

Detector resolution: 8.333 pixels $\mathrm{mm}^{-1}$

$\omega$ scans

Absorption correction: multi-scan

(SADABS; Bruker, 2009)

$T_{\min }=0.853, T_{\max }=0.958$
25410 measured reflections

4757 independent reflections

4316 reflections with $I>2 \sigma(I)$

$R_{\text {int }}=0.022$

$\theta_{\text {max }}=25.0^{\circ}, \theta_{\min }=1.4^{\circ}$

$h=-17 \rightarrow 17$

$k=-19 \rightarrow 19$

$l=-13 \rightarrow 13$ 


\section{Refinement}

Refinement on $F^{2}$

Least-squares matrix: full

$R\left[F^{2}>2 \sigma\left(F^{2}\right)\right]=0.032$

$w R\left(F^{2}\right)=0.086$

$S=1.02$

4757 reflections

375 parameters

30 restraints

Primary atom site location: structure-invariant direct methods
Secondary atom site location: difference Fourier map

Hydrogen site location: inferred from neighbouring sites

$\mathrm{H}$-atom parameters constrained

$w=1 /\left[\sigma^{2}\left(F_{\mathrm{o}}^{2}\right)+(0.040 P)^{2}+1.5222 P\right]$ where $P=\left(F_{\mathrm{o}}{ }^{2}+2 F_{\mathrm{c}}{ }^{2}\right) / 3$

$(\Delta / \sigma)_{\max }<0.001$

$\Delta \rho_{\max }=0.26 \mathrm{e} \AA^{-3}$

$\Delta \rho_{\min }=-0.23$ e $\AA^{-3}$

Special details

Geometry. All e.s.d.'s (except the e.s.d. in the dihedral angle between two l.s. planes) are estimated using the full covariance matrix. The cell e.s.d.'s are taken into account individually in the estimation of e.s.d.'s in distances, angles and torsion angles; correlations between e.s.d.'s in cell parameters are only used when they are defined by crystal symmetry. An approximate (isotropic) treatment of cell e.s.d.'s is used for estimating e.s.d.'s involving 1.s. planes.

Refinement. Refinement of $F^{2}$ against ALL reflections. The weighted $R$-factor $w R$ and goodness of fit $S$ are based on $F^{2}$, conventional $R$-factors $R$ are based on $F$, with $F$ set to zero for negative $F^{2}$. The threshold expression of $F^{2}>\sigma\left(F^{2}\right)$ is used only for calculating $R$-factors(gt) etc. and is not relevant to the choice of reflections for refinement. $R$-factors based on $F^{2}$ are statistically about twice as large as those based on $F$, and $R$ - factors based on ALL data will be even larger.

Fractional atomic coordinates and isotropic or equivalent isotropic displacement parameters $\left(\AA^{2}\right)$

\begin{tabular}{|c|c|c|c|c|c|}
\hline & $x$ & $y$ & $z$ & $U_{\text {iso }} * / U_{\text {eq }}$ & Occ. $(<1)$ \\
\hline $\mathrm{C} 1$ & $0.99995(10)$ & $0.03172(9)$ & $0.34623(12)$ & 0.0231 & \\
\hline $\mathrm{C} 2$ & $1.08898(11)$ & $0.03216(10)$ & $0.40315(13)$ & $0.0282(3)$ & \\
\hline H1 & 1.1151 & 0.0815 & 0.4321 & $0.034 *$ & \\
\hline $\mathrm{C} 3$ & $1.13819(11)$ & $-0.04082(11)$ & $0.41634(14)$ & $0.0316(4)$ & \\
\hline $\mathrm{H} 2$ & 1.197 & -0.0413 & 0.4563 & $0.038^{*}$ & \\
\hline $\mathrm{C} 4$ & $1.09998(11)$ & $-0.11405(11)$ & $0.36986(14)$ & $0.0310(4)$ & \\
\hline H3 & 1.1343 & -0.163 & 0.3759 & $0.037^{*}$ & \\
\hline $\mathrm{C} 5$ & $1.01118(10)$ & $-0.11389(10)$ & $0.31490(13)$ & $0.0257(3)$ & \\
\hline $\mathrm{H} 4$ & 0.9864 & -0.1631 & 0.2837 & $0.031 *$ & \\
\hline C6 & $0.95710(10)$ & $-0.04105(9)$ & $0.30487(12)$ & $0.0208(3)$ & \\
\hline $\mathrm{C} 7$ & $0.86234(9)$ & $-0.03596(9)$ & $0.24765(11)$ & $0.0190(3)$ & \\
\hline $\mathrm{C} 8$ & $0.82094(10)$ & $0.04097(9)$ & $0.23612(12)$ & 0.0199 (3) & \\
\hline C9 & $0.87117(10)$ & $0.11639(9)$ & $0.27233(13)$ & 0.0239 (3) & \\
\hline $\mathrm{C} 10$ & $0.81001(10)$ & $-0.18053(9)$ & $0.27629(12)$ & 0.0215 & \\
\hline H5 & 0.7459 & -0.1825 & 0.2962 & $0.026^{*}$ & \\
\hline H6 & 0.8502 & -0.1745 & 0.3468 & $0.026^{*}$ & \\
\hline C11 & $0.83315(11)$ & $-0.26140(9)$ & $0.21796(12)$ & 0.0242 & \\
\hline $\mathrm{H} 7$ & 0.8264 & -0.3073 & 0.2703 & $0.029 *$ & \\
\hline H8 & 0.8976 & -0.2601 & 0.1993 & $0.029 *$ & \\
\hline $\mathrm{C} 12$ & $0.77517(10)$ & $-0.17887(9)$ & $0.02143(12)$ & 0.0238 & \\
\hline $\mathrm{H} 10$ & 0.8391 & -0.1757 & 0.0011 & $0.029 *$ & \\
\hline H9 & 0.7342 & -0.1748 & -0.0488 & $0.029 *$ & \\
\hline C13 & $0.75641(10)$ & -0.10617 (9) & $0.09892(12)$ & $0.0217(3)$ & \\
\hline H11 & 0.7635 & -0.0545 & 0.0581 & $0.026^{*}$ & \\
\hline $\mathrm{H} 12$ & 0.6928 & -0.1092 & 0.1205 & $0.026^{*}$ & \\
\hline
\end{tabular}




\begin{tabular}{|c|c|c|c|c|c|}
\hline $\mathrm{C} 14$ & $0.72271(10)$ & $0.05441(8)$ & $0.19528(12)$ & 0.0201 & \\
\hline $\mathrm{C} 15$ & $0.64058(9)$ & $0.03168(8)$ & $0.23338(12)$ & 0.0185 & \\
\hline $\mathrm{C} 16$ & $0.56676(10)$ & $0.06269(9)$ & $0.15304(12)$ & $0.0194(3)$ & \\
\hline $\mathrm{C} 17$ & $0.61162(10)$ & $0.10511(9)$ & $0.07106(12)$ & $0.0214(3)$ & \\
\hline $\mathrm{C} 18$ & $0.62518(11)$ & $0.20817(9)$ & $-0.09030(12)$ & $0.0243(3)$ & \\
\hline C19 & $0.68655(12)$ & $0.26826(10)$ & $-0.01648(14)$ & $0.0323(4)$ & \\
\hline H14 & 0.6495 & 0.296 & 0.0363 & $0.048^{*}$ & \\
\hline H16 & 0.7128 & 0.3086 & -0.0648 & $0.048^{*}$ & \\
\hline H15 & 0.736 & 0.2381 & 0.0255 & $0.048^{*}$ & \\
\hline $\mathrm{C} 20$ & $0.54811(12)$ & $0.25574(10)$ & $-0.16032(14)$ & $0.0320(4)$ & \\
\hline H17 & 0.5063 & 0.2172 & -0.2007 & $0.048 *$ & \\
\hline H18 & 0.5754 & 0.2911 & -0.2144 & $0.048 *$ & \\
\hline H19 & 0.5142 & 0.2889 & -0.1099 & $0.048 *$ & \\
\hline $\mathrm{C} 21$ & $0.68273(12)$ & $0.15921(10)$ & -0.17061 & $0.0323(4)$ & \\
\hline $\mathrm{H} 20$ & 0.727 & 0.1249 & -0.1262 & $0.048^{*}$ & \\
\hline $\mathrm{H} 22$ & 0.7154 & 0.197 & -0.2163 & $0.048^{*}$ & \\
\hline $\mathrm{H} 21$ & 0.642 & 0.1249 & -0.2198 & $0.048 *$ & \\
\hline $\mathrm{C} 22$ & $0.46747(10)$ & $0.06935(9)$ & $0.16008(12)$ & $0.0204(3)$ & \\
\hline $\mathrm{C} 23$ & $0.34095(10)$ & $0.05062(10)$ & $0.27252(14)$ & $0.0297(4)$ & \\
\hline $\mathrm{H} 23$ & 0.3276 & 0.1089 & 0.2747 & $0.045^{*}$ & \\
\hline $\mathrm{H} 24$ & 0.3263 & 0.0253 & 0.343 & $0.045^{*}$ & \\
\hline $\mathrm{H} 25$ & 0.3039 & 0.0256 & 0.2095 & $0.045^{*}$ & \\
\hline $\mathrm{C} 24$ & $0.62849(9)$ & $-0.01445(9)$ & $0.34073(12)$ & 0.0200 & \\
\hline $\mathrm{C} 25$ & $0.55129(12)$ & $-0.12568(10)$ & $0.42109(13)$ & 0.0298 & \\
\hline $\mathrm{H} 26$ & 0.605 & -0.1357 & 0.4739 & $0.045^{*}$ & \\
\hline $\mathrm{H} 27$ & 0.522 & -0.1775 & 0.3994 & $0.045^{*}$ & \\
\hline $\mathrm{H} 28$ & 0.5078 & -0.0912 & 0.457 & $0.045^{*}$ & \\
\hline C1S & $1.0683(9)$ & $-0.1031(8)$ & $1.0236(11)$ & $0.059(3)$ & 0.5 \\
\hline H1SA & 1.1073 & -0.0784 & 1.0852 & $0.088^{*}$ & 0.5 \\
\hline H1SB & 1.1057 & -0.1181 & 0.9628 & $0.088 *$ & 0.5 \\
\hline H1SC & 1.0388 & -0.1517 & 1.0511 & $0.088^{*}$ & 0.5 \\
\hline $\mathrm{C} 2 \mathrm{~S}$ & $0.994(2)$ & $-0.041(2)$ & $0.979(2)$ & $0.0624(17)$ & 0.5 \\
\hline $\mathrm{C} 3 \mathrm{~S}$ & $0.9217(9)$ & $0.0898(7)$ & $0.9635(11)$ & $0.0506(17)$ & 0.5 \\
\hline H3SA & 0.8679 & 0.0739 & 1.0031 & $0.061 *$ & 0.5 \\
\hline H3SB & 0.9073 & 0.0815 & 0.8817 & $0.061 *$ & 0.5 \\
\hline $\mathrm{C} 4 \mathrm{~S}$ & $0.9519(2)$ & $0.1897(2)$ & $0.9924(3)$ & $0.0399(9)$ & 0.5 \\
\hline H4SA & 0.9024 & 0.2257 & 0.9627 & $0.06^{*}$ & 0.5 \\
\hline $\mathrm{H} 4 \mathrm{SB}$ & 1.0079 & 0.2028 & 0.9569 & $0.06^{*}$ & 0.5 \\
\hline $\mathrm{H} 4 \mathrm{SC}$ & 0.9627 & 0.1971 & 1.0738 & $0.06 *$ & 0.5 \\
\hline N1 & $0.82204(8)$ & $-0.10814(7)$ & $0.20229(10)$ & $0.0197(3)$ & \\
\hline N2 & $0.57584(9)$ & $0.15043(8)$ & $-0.01773(11)$ & $0.0286(3)$ & \\
\hline H13 & 0.5167 & 0.1455 & -0.0349 & $0.034 *$ & \\
\hline $\mathrm{O} 1$ & $0.95818(7)$ & $0.10811(6)$ & $0.32980(9)$ & $0.0263(2)$ & \\
\hline $\mathrm{O} 2$ & $0.84156(8)$ & $0.18618(7)$ & $0.25827(10)$ & $0.0324(3)$ & \\
\hline $\mathrm{O} 3$ & $0.70539(7)$ & $0.09935(6)$ & $0.09266(8)$ & $0.0223(2)$ & \\
\hline O4 & $0.41429(7)$ & $0.10217(7)$ & $0.08665(9)$ & $0.0299(3)$ & \\
\hline $\mathrm{O5}$ & $0.43880(7)$ & $0.03856(6)$ & $0.25799(8)$ & $0.0226(2)$ & \\
\hline O6 & $0.65724(8)$ & $0.00853(8)$ & $0.43480(9)$ & $0.0370(3)$ & \\
\hline
\end{tabular}




\begin{tabular}{llllll} 
O7 & $0.57991(7)$ & $-0.08439(6)$ & $0.32009(8)$ & $0.0235(2)$ & \\
O1S & $0.9264(3)$ & $-0.0628(3)$ & $0.9137(4)$ & $0.1025(13)$ & 0.5 \\
O2S & $1.0034(13)$ & $0.0428(14)$ & $1.0052(12)$ & $0.0550(15)$ & 0.5 \\
S1 & $0.75716(3)$ & $-0.27772(2)$ & $0.08816(3)$ & $0.02791(11)$ & \\
\hline
\end{tabular}

Atomic displacement parameters $\left(\AA^{2}\right)$

\begin{tabular}{|c|c|c|c|c|c|c|}
\hline & $U^{11}$ & $U^{22}$ & $U^{33}$ & $U^{12}$ & $U^{13}$ & $U^{23}$ \\
\hline $\mathrm{C} 1$ & $0.0205(7)$ & $0.0277(8)$ & $0.0213(7)$ & $-0.0025(6)$ & $0.0037(6)$ & $0.0008(6)$ \\
\hline $\mathrm{C} 2$ & $0.0218(8)$ & $0.0373(9)$ & $0.0252(8)$ & $-0.0098(7)$ & $-0.0001(6)$ & $-0.0009(7)$ \\
\hline $\mathrm{C} 3$ & $0.0166(7)$ & $0.0500(10)$ & $0.0272(8)$ & $-0.0029(7)$ & $-0.0034(6)$ & $0.0055(7)$ \\
\hline $\mathrm{C} 4$ & $0.0216(8)$ & $0.0390(9)$ & $0.0317(9)$ & $0.0058(7)$ & $-0.0020(6)$ & $0.0040(7)$ \\
\hline $\mathrm{C} 5$ & $0.0219(7)$ & $0.0290(8)$ & $0.0256(8)$ & $0.0008(6)$ & $-0.0010(6)$ & $0.0000(6)$ \\
\hline C6 & $0.0177(7)$ & $0.0277(8)$ & $0.0171(7)$ & $-0.0014(6)$ & $0.0015(5)$ & $0.0016(6)$ \\
\hline $\mathrm{C} 7$ & 0.0177 (7) & $0.0236(7)$ & $0.0159(7)$ & $-0.0013(6)$ & $0.0026(5)$ & $0.0023(5)$ \\
\hline $\mathrm{C} 8$ & $0.0178(7)$ & $0.0231(7)$ & $0.0191(7)$ & $-0.0012(6)$ & $0.0031(5)$ & $0.0015(6)$ \\
\hline C9 & $0.0219(7)$ & $0.0257(8)$ & $0.0245(8)$ & $-0.0017(6)$ & $0.0043(6)$ & $0.0002(6)$ \\
\hline $\mathrm{C} 10$ & $0.0219(7)$ & $0.0227(7)$ & $0.0194(7)$ & $-0.0020(6)$ & $-0.0001(6)$ & $0.0041(6)$ \\
\hline C11 & $0.0270(8)$ & $0.0221(7)$ & $0.0229(7)$ & $-0.0015(6)$ & $-0.0020(6)$ & $0.0043(6)$ \\
\hline $\mathrm{C} 12$ & $0.0228(7)$ & $0.0283(8)$ & $0.0194(7)$ & $0.0022(6)$ & $-0.0035(6)$ & $0.0008(6)$ \\
\hline $\mathrm{C} 13$ & $0.0210(7)$ & $0.0228(7)$ & $0.0202(7)$ & $0.0006(6)$ & $-0.0046(6)$ & $0.0018(6)$ \\
\hline $\mathrm{C} 14$ & $0.0224(7)$ & $0.0175(7)$ & $0.0200(7)$ & $0.0018(6)$ & $0.0002(6)$ & $0.0034(5)$ \\
\hline $\mathrm{C} 15$ & $0.0191(7)$ & $0.0168(7)$ & $0.0194(7)$ & $0.0016(5)$ & $0.0004(5)$ & $-0.0004(5)$ \\
\hline $\mathrm{C} 16$ & $0.0189(7)$ & $0.0200(7)$ & $0.0187(7)$ & $0.0018(5)$ & $-0.0008(5)$ & $0.0010(5)$ \\
\hline $\mathrm{C} 17$ & $0.0195(7)$ & $0.0226(7)$ & $0.0218(7)$ & $0.0028(6)$ & $-0.0004(6)$ & $0.0019(6)$ \\
\hline $\mathrm{C} 18$ & $0.0309(8)$ & $0.0213(7)$ & $0.0209(7)$ & $0.0007(6)$ & $0.0030(6)$ & $0.0039(6)$ \\
\hline C19 & $0.0402(9)$ & $0.0246(8)$ & $0.0314(9)$ & $-0.0006(7)$ & $-0.0010(7)$ & $0.0005(7)$ \\
\hline $\mathrm{C} 20$ & $0.0425(10)$ & $0.0277(8)$ & $0.0251(8)$ & $0.0061(7)$ & $-0.0007(7)$ & $0.0049(6)$ \\
\hline $\mathrm{C} 21$ & $0.0378(9)$ & $0.0299(8)$ & $0.0300(8)$ & $0.0010(7)$ & $0.0075(7)$ & $-0.0007(7)$ \\
\hline $\mathrm{C} 22$ & $0.0203(7)$ & $0.0194(7)$ & $0.0209(7)$ & $0.0005(6)$ & $-0.0003(6)$ & $-0.0014(6)$ \\
\hline $\mathrm{C} 23$ & $0.0207(8)$ & $0.0340(9)$ & $0.0353(9)$ & $0.0037(6)$ & $0.0082(6)$ & $0.0009(7)$ \\
\hline $\mathrm{C} 24$ & $0.0148(6)$ & $0.0229(7)$ & $0.0219(7)$ & $0.0010(5)$ & $-0.0006(5)$ & $0.0021(6)$ \\
\hline $\mathrm{C} 25$ & $0.0328(9)$ & $0.0313(8)$ & $0.0249(8)$ & $-0.0065(7)$ & $0.0008(6)$ & $0.0099(7)$ \\
\hline $\mathrm{C} 1 \mathrm{~S}$ & $0.036(4)$ & $0.090(5)$ & $0.055(4)$ & $-0.004(3)$ & $0.028(3)$ & $-0.002(3)$ \\
\hline $\mathrm{C} 2 \mathrm{~S}$ & 0.049 (3) & $0.082(3)$ & $0.060(4)$ & $-0.003(2)$ & $0.026(3)$ & 0.013 \\
\hline $\mathrm{C} 3 \mathrm{~S}$ & $0.036(3)$ & $0.070(3)$ & $0.047(3)$ & $0.013(3)$ & $0.007(2)$ & $0.020(3)$ \\
\hline $\mathrm{C} 4 \mathrm{~S}$ & $0.0258(17)$ & $0.052(2)$ & $0.044(2)$ & $0.0120(15)$ & $0.0153(15)$ & $0.0192(17)$ \\
\hline N1 & $0.0199(6)$ & $0.0199(6)$ & $0.0185(6)$ & $-0.0012(5)$ & $-0.0039(5)$ & $0.0028(5)$ \\
\hline N2 & $0.0200(6)$ & $0.0372(8)$ & $0.0280(7)$ & $0.0004(5)$ & $-0.0007(5)$ & $0.0136(6)$ \\
\hline $\mathrm{O} 1$ & $0.0223(5)$ & $0.0244(5)$ & $0.0319(6)$ & -0.0049 & 0.0000 & -0.0021 \\
\hline $\mathrm{O} 2$ & $0.0326(6)$ & $0.0207(6)$ & $0.0437(7)$ & $0.0006(5)$ & $0.0019(5)$ & $-0.0024(5)$ \\
\hline $\mathrm{O} 3$ & $0.0185(5)$ & $0.0251(5)$ & $0.0232(5)$ & $0.0016(4)$ & $0.0023(4)$ & $0.0073(4)$ \\
\hline $\mathrm{O} 4$ & $0.0204(5)$ & $0.0408(7)$ & $0.0277(6)$ & $0.0047(5)$ & $-0.0027(4)$ & $0.0086(5)$ \\
\hline O5 & $0.0175(5)$ & $0.0271(5)$ & $0.0236(5)$ & 0.0021 & 0.0035 (4) & $0.0028(4)$ \\
\hline O6 & $0.0417(7)$ & $0.0463(7)$ & $0.0214(6)$ & $-0.0190(6)$ & $-0.0069(5)$ & $0.0048(5)$ \\
\hline $\mathrm{O} 7$ & $0.0284(5)$ & $0.0218(5)$ & $0.0202(5)$ & -0.0045 & 0.0014 (4) & 0.0039 (4) \\
\hline O1S & $0.104(3)$ & 0.113 & 0.094 (3) & -0.019 & $0.029(2)$ & $0.021(3)$ \\
\hline $\mathrm{O} 2 \mathrm{~S}$ & $0.0419(18)$ & $0.0739(19)$ & $0.052(4)$ & $0.0088(17)$ & $0.017(3)$ & $0.014(3)$ \\
\hline
\end{tabular}




$\mathrm{S} 1 \quad 0.0304(2) \quad 0.0225(2) \quad 0.0294(2) \quad-0.00151(15) \quad-0.00565(16) \quad-0.00288(15)$

Geometric parameters $(\AA, \stackrel{o}{)}$

\begin{tabular}{|c|c|c|c|}
\hline $\mathrm{C} 1-\mathrm{O} 1$ & $1.3759(18)$ & $\mathrm{C} 18-\mathrm{N} 2$ & $1.4817(19)$ \\
\hline $\mathrm{C} 1-\mathrm{C} 2$ & $1.390(2)$ & $\mathrm{C} 18-\mathrm{C} 21$ & $1.525(2)$ \\
\hline $\mathrm{C} 1-\mathrm{C} 6$ & $1.392(2)$ & $\mathrm{C} 18-\mathrm{C} 20$ & $1.526(2)$ \\
\hline $\mathrm{C} 2-\mathrm{C} 3$ & $1.374(2)$ & $\mathrm{C} 18-\mathrm{C} 19$ & $1.526(2)$ \\
\hline $\mathrm{C} 2-\mathrm{H} 1$ & 0.93 & C19-H14 & 0.96 \\
\hline $\mathrm{C} 3-\mathrm{C} 4$ & $1.392(2)$ & C19-H16 & 0.96 \\
\hline $\mathrm{C} 3-\mathrm{H} 2$ & 0.93 & $\mathrm{C} 19-\mathrm{H} 15$ & 0.96 \\
\hline $\mathrm{C} 4-\mathrm{C} 5$ & $1.378(2)$ & $\mathrm{C} 20-\mathrm{H} 17$ & 0.96 \\
\hline $\mathrm{C} 4-\mathrm{H} 3$ & 0.93 & $\mathrm{C} 20-\mathrm{H} 18$ & 0.96 \\
\hline $\mathrm{C} 5-\mathrm{C} 6$ & $1.407(2)$ & $\mathrm{C} 20-\mathrm{H} 19$ & 0.96 \\
\hline $\mathrm{C} 5-\mathrm{H} 4$ & 0.93 & $\mathrm{C} 21-\mathrm{H} 20$ & 0.96 \\
\hline $\mathrm{C} 6-\mathrm{C} 7$ & $1.4667(19)$ & $\mathrm{C} 21-\mathrm{H} 22$ & 0.96 \\
\hline $\mathrm{C} 7-\mathrm{C} 8$ & $1.377(2)$ & $\mathrm{C} 21-\mathrm{H} 21$ & 0.96 \\
\hline $\mathrm{C} 7-\mathrm{N} 1$ & $1.3844(18)$ & $\mathrm{C} 22-\mathrm{O} 4$ & $1.2197(17)$ \\
\hline $\mathrm{C} 8-\mathrm{C} 9$ & $1.458(2)$ & $\mathrm{C} 22-\mathrm{O} 5$ & 1.3457 (17) \\
\hline $\mathrm{C} 8-\mathrm{C} 14$ & $1.467(2)$ & $\mathrm{C} 23-\mathrm{O} 5$ & $1.4444(17)$ \\
\hline $\mathrm{C} 9-\mathrm{O} 2$ & $1.2088(19)$ & $\mathrm{C} 23-\mathrm{H} 23$ & 0.96 \\
\hline $\mathrm{C} 9-\mathrm{O} 1$ & $1.3737(18)$ & $\mathrm{C} 23-\mathrm{H} 24$ & 0.96 \\
\hline $\mathrm{C} 10-\mathrm{N} 1$ & $1.4721(18)$ & $\mathrm{C} 23-\mathrm{H} 25$ & 0.96 \\
\hline $\mathrm{C} 10-\mathrm{C} 11$ & $1.521(2)$ & $\mathrm{C} 24-\mathrm{O} 6$ & $1.2001(18)$ \\
\hline $\mathrm{C} 10-\mathrm{H} 5$ & 0.97 & $\mathrm{C} 24-\mathrm{O} 7$ & $1.3367(17)$ \\
\hline $\mathrm{C} 10-\mathrm{H} 6$ & 0.97 & $\mathrm{C} 25-\mathrm{O} 7$ & $1.4469(18)$ \\
\hline $\mathrm{C} 11-\mathrm{S} 1$ & $1.8110(15)$ & $\mathrm{C} 25-\mathrm{H} 26$ & 0.96 \\
\hline $\mathrm{C} 11-\mathrm{H} 7$ & 0.97 & $\mathrm{C} 25-\mathrm{H} 27$ & 0.96 \\
\hline $\mathrm{C} 11-\mathrm{H} 8$ & 0.97 & $\mathrm{C} 25-\mathrm{H} 28$ & 0.96 \\
\hline $\mathrm{C} 12-\mathrm{C} 13$ & $1.520(2)$ & $\mathrm{C} 1 \mathrm{~S}-\mathrm{C} 2 \mathrm{~S}$ & $1.51(3)$ \\
\hline $\mathrm{C} 12-\mathrm{S} 1$ & $1.8027(15)$ & C1S-H1SA & 0.96 \\
\hline $\mathrm{C} 12-\mathrm{H} 10$ & 0.97 & C1S-H1SB & 0.96 \\
\hline $\mathrm{C} 12-\mathrm{H} 9$ & 0.97 & $\mathrm{C} 1 \mathrm{~S}-\mathrm{H} 1 \mathrm{SC}$ & 0.96 \\
\hline $\mathrm{C} 13-\mathrm{N} 1$ & $1.4680(17)$ & $\mathrm{C} 2 \mathrm{~S}-\mathrm{O} 1 \mathrm{~S}$ & $1.23(3)$ \\
\hline C13-H11 & 0.97 & $\mathrm{C} 2 \mathrm{~S}-\mathrm{O} 2 \mathrm{~S}$ & $1.396(16)$ \\
\hline $\mathrm{C} 13-\mathrm{H} 12$ & 0.97 & $\mathrm{C} 3 \mathrm{~S}-\mathrm{O} 2 \mathrm{~S}$ & $1.446(19)$ \\
\hline $\mathrm{C} 14-\mathrm{C} 15$ & $1.348(2)$ & $\mathrm{C} 3 \mathrm{~S}-\mathrm{C} 4 \mathrm{~S}$ & $1.693(13)$ \\
\hline $\mathrm{C} 14-\mathrm{O} 3$ & $1.4058(17)$ & $\mathrm{C} 3 \mathrm{~S}-\mathrm{H} 3 \mathrm{SA}$ & 0.97 \\
\hline $\mathrm{C} 15-\mathrm{C} 16$ & $1.4440(19)$ & $\mathrm{C} 3 \mathrm{~S}-\mathrm{H} 3 \mathrm{SB}$ & 0.97 \\
\hline $\mathrm{C} 15-\mathrm{C} 24$ & $1.4830(19)$ & $\mathrm{C} 4 \mathrm{~S}-\mathrm{H} 4 \mathrm{SA}$ & 0.96 \\
\hline $\mathrm{C} 16-\mathrm{C} 17$ & $1.383(2)$ & $\mathrm{C} 4 \mathrm{~S}-\mathrm{H} 4 \mathrm{SB}$ & 0.96 \\
\hline $\mathrm{C} 16-\mathrm{C} 22$ & $1.441(2)$ & $\mathrm{C} 4 \mathrm{~S}-\mathrm{H} 4 \mathrm{SC}$ & 0.96 \\
\hline $\mathrm{C} 17-\mathrm{N} 2$ & $1.3357(19)$ & $\mathrm{N} 2-\mathrm{H} 13$ & 0.86 \\
\hline $\mathrm{C} 17-\mathrm{O} 3$ & $1.3527(17)$ & & \\
\hline $\mathrm{O} 1-\mathrm{C} 1-\mathrm{C} 2$ & $115.75(13)$ & $\mathrm{C} 18-\mathrm{C} 19-\mathrm{H} 14$ & 109.5 \\
\hline $\mathrm{O} 1-\mathrm{C} 1-\mathrm{C} 6$ & $122.08(13)$ & $\mathrm{C} 18-\mathrm{C} 19-\mathrm{H} 16$ & 109.5 \\
\hline $\mathrm{C} 2-\mathrm{C} 1-\mathrm{C} 6$ & $122.13(14)$ & $\mathrm{H} 14-\mathrm{C} 19-\mathrm{H} 16$ & 109.5 \\
\hline
\end{tabular}




\begin{tabular}{|c|c|c|c|}
\hline $\mathrm{C} 3-\mathrm{C} 2-\mathrm{C} 1$ & $119.52(15)$ & $\mathrm{C} 18-\mathrm{C} 19-\mathrm{H} 15$ & 109.5 \\
\hline $\mathrm{C} 3-\mathrm{C} 2-\mathrm{H} 1$ & 120.2 & $\mathrm{H} 14-\mathrm{C} 19-\mathrm{H} 15$ & 109.5 \\
\hline $\mathrm{C} 1-\mathrm{C} 2-\mathrm{H} 1$ & 120.2 & $\mathrm{H} 16-\mathrm{C} 19-\mathrm{H} 15$ & 109.5 \\
\hline $\mathrm{C} 2-\mathrm{C} 3-\mathrm{C} 4$ & $120.00(14)$ & $\mathrm{C} 18-\mathrm{C} 20-\mathrm{H} 17$ & 109.5 \\
\hline $\mathrm{C} 2-\mathrm{C} 3-\mathrm{H} 2$ & 120.0 & $\mathrm{C} 18-\mathrm{C} 20-\mathrm{H} 18$ & 109.5 \\
\hline $\mathrm{C} 4-\mathrm{C} 3-\mathrm{H} 2$ & 120.0 & $\mathrm{H} 17-\mathrm{C} 20-\mathrm{H} 18$ & 109.5 \\
\hline $\mathrm{C} 5-\mathrm{C} 4-\mathrm{C} 3$ & $119.90(15)$ & $\mathrm{C} 18-\mathrm{C} 20-\mathrm{H} 19$ & 109.5 \\
\hline $\mathrm{C} 5-\mathrm{C} 4-\mathrm{H} 3$ & 120.1 & $\mathrm{H} 17-\mathrm{C} 20-\mathrm{H} 19$ & 109.5 \\
\hline $\mathrm{C} 3-\mathrm{C} 4-\mathrm{H} 3$ & 120.1 & $\mathrm{H} 18-\mathrm{C} 20-\mathrm{H} 19$ & 109.5 \\
\hline $\mathrm{C} 4-\mathrm{C} 5-\mathrm{C} 6$ & $121.52(15)$ & $\mathrm{C} 18-\mathrm{C} 21-\mathrm{H} 20$ & 109.5 \\
\hline $\mathrm{C} 4-\mathrm{C} 5-\mathrm{H} 4$ & 119.2 & $\mathrm{C} 18-\mathrm{C} 21-\mathrm{H} 22$ & 109.5 \\
\hline $\mathrm{C} 6-\mathrm{C} 5-\mathrm{H} 4$ & 119.2 & $\mathrm{H} 20-\mathrm{C} 21-\mathrm{H} 22$ & 109.5 \\
\hline $\mathrm{C} 1-\mathrm{C} 6-\mathrm{C} 5$ & $116.71(13)$ & $\mathrm{C} 18-\mathrm{C} 21-\mathrm{H} 21$ & 109.5 \\
\hline $\mathrm{C} 1-\mathrm{C} 6-\mathrm{C} 7$ & $118.48(13)$ & $\mathrm{H} 20-\mathrm{C} 21-\mathrm{H} 21$ & 109.5 \\
\hline $\mathrm{C} 5-\mathrm{C} 6-\mathrm{C} 7$ & $124.67(13)$ & $\mathrm{H} 22-\mathrm{C} 21-\mathrm{H} 21$ & 109.5 \\
\hline $\mathrm{C} 8-\mathrm{C} 7-\mathrm{N} 1$ & $123.80(12)$ & $\mathrm{O} 4-\mathrm{C} 22-\mathrm{O} 5$ & $122.69(13)$ \\
\hline $\mathrm{C} 8-\mathrm{C} 7-\mathrm{C} 6$ & $118.12(13)$ & $\mathrm{O} 4-\mathrm{C} 22-\mathrm{C} 16$ & $123.71(13)$ \\
\hline $\mathrm{N} 1-\mathrm{C} 7-\mathrm{C} 6$ & $117.96(12)$ & $\mathrm{O} 5-\mathrm{C} 22-\mathrm{C} 16$ & $113.56(12)$ \\
\hline $\mathrm{C} 7-\mathrm{C} 8-\mathrm{C} 9$ & $121.56(13)$ & $\mathrm{O} 5-\mathrm{C} 23-\mathrm{H} 23$ & 109.5 \\
\hline $\mathrm{C} 7-\mathrm{C} 8-\mathrm{C} 14$ & $124.09(13)$ & $\mathrm{O} 5-\mathrm{C} 23-\mathrm{H} 24$ & 109.5 \\
\hline $\mathrm{C} 9-\mathrm{C} 8-\mathrm{C} 14$ & $114.21(12)$ & $\mathrm{H} 23-\mathrm{C} 23-\mathrm{H} 24$ & 109.5 \\
\hline $\mathrm{O} 2-\mathrm{C} 9-\mathrm{O} 1$ & $116.85(13)$ & $\mathrm{O} 5-\mathrm{C} 23-\mathrm{H} 25$ & 109.5 \\
\hline $\mathrm{O} 2-\mathrm{C} 9-\mathrm{C} 8$ & $125.21(14)$ & $\mathrm{H} 23-\mathrm{C} 23-\mathrm{H} 25$ & 109.5 \\
\hline $\mathrm{O} 1-\mathrm{C} 9-\mathrm{C} 8$ & $117.91(12)$ & $\mathrm{H} 24-\mathrm{C} 23-\mathrm{H} 25$ & 109.5 \\
\hline $\mathrm{N} 1-\mathrm{C} 10-\mathrm{C} 11$ & $111.94(11)$ & $\mathrm{O} 6-\mathrm{C} 24-\mathrm{O} 7$ & $123.96(13)$ \\
\hline $\mathrm{N} 1-\mathrm{C} 10-\mathrm{H} 5$ & 109.2 & $\mathrm{O} 6-\mathrm{C} 24-\mathrm{C} 15$ & $124.46(13)$ \\
\hline $\mathrm{C} 11-\mathrm{C} 10-\mathrm{H} 5$ & 109.2 & $\mathrm{O} 7-\mathrm{C} 24-\mathrm{C} 15$ & 111.57 (12) \\
\hline $\mathrm{N} 1-\mathrm{C} 10-\mathrm{H} 6$ & 109.2 & $\mathrm{O} 7-\mathrm{C} 25-\mathrm{H} 26$ & 109.5 \\
\hline $\mathrm{C} 11-\mathrm{C} 10-\mathrm{H} 6$ & 109.2 & $\mathrm{O} 7-\mathrm{C} 25-\mathrm{H} 27$ & 109.5 \\
\hline $\mathrm{H} 5-\mathrm{C} 10-\mathrm{H} 6$ & 107.9 & $\mathrm{H} 26-\mathrm{C} 25-\mathrm{H} 27$ & 109.5 \\
\hline $\mathrm{C} 10-\mathrm{C} 11-\mathrm{S} 1$ & $111.17(10)$ & $\mathrm{O} 7-\mathrm{C} 25-\mathrm{H} 28$ & 109.5 \\
\hline $\mathrm{C} 10-\mathrm{C} 11-\mathrm{H} 7$ & 109.4 & $\mathrm{H} 26-\mathrm{C} 25-\mathrm{H} 28$ & 109.5 \\
\hline $\mathrm{S} 1-\mathrm{C} 11-\mathrm{H} 7$ & 109.4 & $\mathrm{H} 27-\mathrm{C} 25-\mathrm{H} 28$ & 109.5 \\
\hline $\mathrm{C} 10-\mathrm{C} 11-\mathrm{H} 8$ & 109.4 & $\mathrm{C} 2 \mathrm{~S}-\mathrm{C} 1 \mathrm{~S}-\mathrm{H} 1 \mathrm{SA}$ & 109.5 \\
\hline $\mathrm{S} 1-\mathrm{C} 11-\mathrm{H} 8$ & 109.4 & $\mathrm{C} 2 \mathrm{~S}-\mathrm{C} 1 \mathrm{~S}-\mathrm{H} 1 \mathrm{SB}$ & 109.5 \\
\hline $\mathrm{H} 7-\mathrm{C} 11-\mathrm{H} 8$ & 108.0 & $\mathrm{H} 1 \mathrm{SA}-\mathrm{C} 1 \mathrm{~S}-\mathrm{H} 1 \mathrm{SB}$ & 109.5 \\
\hline $\mathrm{C} 13-\mathrm{C} 12-\mathrm{S} 1$ & $112.52(10)$ & $\mathrm{C} 2 \mathrm{~S}-\mathrm{C} 1 \mathrm{~S}-\mathrm{H} 1 \mathrm{SC}$ & 109.5 \\
\hline $\mathrm{C} 13-\mathrm{C} 12-\mathrm{H} 10$ & 109.1 & $\mathrm{H} 1 \mathrm{SA}-\mathrm{C} 1 \mathrm{~S}-\mathrm{H} 1 \mathrm{SC}$ & 109.5 \\
\hline $\mathrm{S} 1-\mathrm{C} 12-\mathrm{H} 10$ & 109.1 & $\mathrm{H} 1 \mathrm{SB}-\mathrm{C} 1 \mathrm{~S}-\mathrm{H} 1 \mathrm{SC}$ & 109.5 \\
\hline $\mathrm{C} 13-\mathrm{C} 12-\mathrm{H} 9$ & 109.1 & $\mathrm{O} 1 \mathrm{~S}-\mathrm{C} 2 \mathrm{~S}-\mathrm{O} 2 \mathrm{~S}$ & $117(2)$ \\
\hline $\mathrm{S} 1-\mathrm{C} 12-\mathrm{H} 9$ & 109.1 & $\mathrm{O} 1 \mathrm{~S}-\mathrm{C} 2 \mathrm{~S}-\mathrm{C} 1 \mathrm{~S}$ & $122(3)$ \\
\hline $\mathrm{H} 10-\mathrm{C} 12-\mathrm{H} 9$ & 107.8 & $\mathrm{O} 2 \mathrm{~S}-\mathrm{C} 2 \mathrm{~S}-\mathrm{C} 1 \mathrm{~S}$ & $120.8(16)$ \\
\hline $\mathrm{N} 1-\mathrm{C} 13-\mathrm{C} 12$ & $109.89(11)$ & $\mathrm{O} 2 \mathrm{~S}-\mathrm{C} 3 \mathrm{~S}-\mathrm{C} 4 \mathrm{~S}$ & $104.1(11)$ \\
\hline $\mathrm{N} 1-\mathrm{C} 13-\mathrm{H} 11$ & 109.7 & $\mathrm{O} 2 \mathrm{~S}-\mathrm{C} 3 \mathrm{~S}-\mathrm{H} 3 \mathrm{SA}$ & 110.9 \\
\hline $\mathrm{C} 12-\mathrm{C} 13-\mathrm{H} 11$ & 109.7 & $\mathrm{C} 4 \mathrm{~S}-\mathrm{C} 3 \mathrm{~S}-\mathrm{H} 3 \mathrm{SA}$ & 110.9 \\
\hline $\mathrm{N} 1-\mathrm{C} 13-\mathrm{H} 12$ & 109.7 & $\mathrm{O} 2 \mathrm{~S}-\mathrm{C} 3 \mathrm{~S}-\mathrm{H} 3 \mathrm{SB}$ & 110.9 \\
\hline $\mathrm{C} 12-\mathrm{C} 13-\mathrm{H} 12$ & 109.7 & $\mathrm{C} 4 \mathrm{~S}-\mathrm{C} 3 \mathrm{~S}-\mathrm{H} 3 \mathrm{SB}$ & 110.9 \\
\hline $\mathrm{H} 11-\mathrm{C} 13-\mathrm{H} 12$ & 108.2 & $\mathrm{H} 3 \mathrm{SA}-\mathrm{C} 3 \mathrm{~S}-\mathrm{H} 3 \mathrm{SB}$ & 109.0 \\
\hline
\end{tabular}




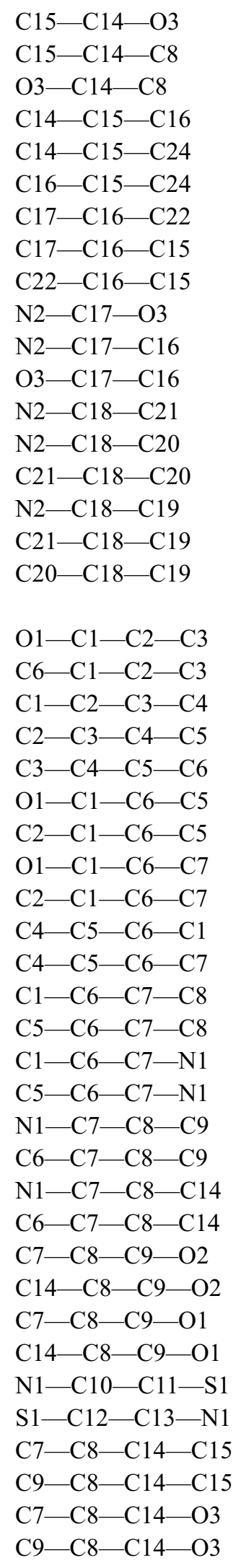

$109.12(12)$

$134.25(13)$

$116.62(12)$

$107.78(12)$

$125.94(13)$

$126.25(12)$

$121.81(13)$

$105.20(12)$

$131.59(13)$

$119.51(13)$

$129.71(13)$

$110.72(12)$

$109.92(13)$

$105.19(12)$

$109.66(13)$

$110.86(12)$

$111.06(13)$

$109.99(13)$

$-175.67(13)$

$2.1(2)$

$1.9(2)$

$-2.8(2)$

$-0.4(2)$

$172.56(13)$

$-5.1(2)$

$-3.4(2)$

$178.92(13)$

$4.2(2)$

$179.92(14)$

0.98 (19)

$-174.66(13)$

$177.15(12)$

1.5 (2)

$-171.86(13)$

4.1 (2)

12.7 (2)

$-171.36(12)$

175.03 (15)

-9.1 (2)

$-6.8(2)$

$169.05(12)$

$-60.96(14)$

62.57 (14)

$61.3(2)$

$-114.41(18)$

$-117.28(15)$

$66.99(16)$

\begin{tabular}{|c|c|}
\hline $\mathrm{C} 3 \mathrm{~S}-\mathrm{C} 4 \mathrm{~S}-\mathrm{H} 4 \mathrm{SA}$ & 109.5 \\
\hline $\mathrm{C} 3 \mathrm{~S}-\mathrm{C} 4 \mathrm{~S}-\mathrm{H} 4 \mathrm{SB}$ & 109.5 \\
\hline $\mathrm{H} 4 \mathrm{SA}-\mathrm{C} 4 \mathrm{~S}-\mathrm{H} 4 \mathrm{SB}$ & 109.5 \\
\hline $\mathrm{C} 3 \mathrm{~S}-\mathrm{C} 4 \mathrm{~S}-\mathrm{H} 4 \mathrm{SC}$ & 109.5 \\
\hline $\mathrm{H} 4 \mathrm{SA}-\mathrm{C} 4 \mathrm{~S}-\mathrm{H} 4 \mathrm{SC}$ & 109.5 \\
\hline $\mathrm{H} 4 \mathrm{SB}-\mathrm{C} 4 \mathrm{~S}-\mathrm{H} 4 \mathrm{SC}$ & 109.5 \\
\hline $\mathrm{C} 7-\mathrm{N} 1-\mathrm{C} 13$ & $121.00(11)$ \\
\hline $\mathrm{C} 7-\mathrm{N} 1-\mathrm{C} 10$ & $120.47(11)$ \\
\hline $\mathrm{C} 13-\mathrm{N} 1-\mathrm{C} 10$ & $113.69(11)$ \\
\hline $\mathrm{C} 17-\mathrm{N} 2-\mathrm{C} 18$ & $128.18(13)$ \\
\hline $\mathrm{C} 17-\mathrm{N} 2-\mathrm{H} 13$ & 115.9 \\
\hline $\mathrm{C} 18-\mathrm{N} 2-\mathrm{H} 13$ & 115.9 \\
\hline $\mathrm{C} 9-\mathrm{O} 1-\mathrm{C} 1$ & $121.51(11)$ \\
\hline $\mathrm{C} 17-\mathrm{O} 3-\mathrm{C} 14$ & $107.11(11)$ \\
\hline $\mathrm{C} 22-\mathrm{O} 5-\mathrm{C} 23$ & $115.10(11)$ \\
\hline $\mathrm{C} 24-\mathrm{O} 7-\mathrm{C} 25$ & $114.92(11)$ \\
\hline $\mathrm{C} 2 \mathrm{~S}-\mathrm{O} 2 \mathrm{~S}-\mathrm{C} 3 \mathrm{~S}$ & $112.1(10)$ \\
\hline $\mathrm{C} 12-\mathrm{S} 1-\mathrm{C} 11$ & $97.82(7)$ \\
\hline $\mathrm{C} 15-\mathrm{C} 16-\mathrm{C} 17-\mathrm{O} 3$ & $2.48(16)$ \\
\hline $\mathrm{C} 17-\mathrm{C} 16-\mathrm{C} 22-\mathrm{O} 4$ & $10.8(2)$ \\
\hline $\mathrm{C} 15-\mathrm{C} 16-\mathrm{C} 22-\mathrm{O} 4$ & $175.22(15)$ \\
\hline $\mathrm{C} 17-\mathrm{C} 16-\mathrm{C} 22-\mathrm{O} 5$ & $-166.81(13)$ \\
\hline $\mathrm{C} 15-\mathrm{C} 16-\mathrm{C} 22-\mathrm{O} 5$ & $-2.4(2)$ \\
\hline $\mathrm{C} 14-\mathrm{C} 15-\mathrm{C} 24-\mathrm{O} 6$ & $58.0(2)$ \\
\hline $\mathrm{C} 16-\mathrm{C} 15-\mathrm{C} 24-\mathrm{O} 6$ & $-119.79(17)$ \\
\hline $\mathrm{C} 14-\mathrm{C} 15-\mathrm{C} 24-\mathrm{O} 7$ & $-123.19(15)$ \\
\hline $\mathrm{C} 16-\mathrm{C} 15-\mathrm{C} 24-\mathrm{O} 7$ & $59.05(18)$ \\
\hline $\mathrm{C} 8-\mathrm{C} 7-\mathrm{N} 1-\mathrm{C} 13$ & $28.2(2)$ \\
\hline $\mathrm{C} 6-\mathrm{C} 7-\mathrm{N} 1-\mathrm{C} 13$ & $-147.75(13)$ \\
\hline $\mathrm{C} 8-\mathrm{C} 7-\mathrm{N} 1-\mathrm{C} 10$ & $-125.64(15)$ \\
\hline $\mathrm{C} 6-\mathrm{C} 7-\mathrm{N} 1-\mathrm{C} 10$ & $58.43(17)$ \\
\hline $\mathrm{C} 12-\mathrm{C} 13-\mathrm{N} 1-\mathrm{C} 7$ & $139.30(13)$ \\
\hline $\mathrm{C} 12-\mathrm{C} 13-\mathrm{N} 1-\mathrm{C} 10$ & $-65.24(15)$ \\
\hline $\mathrm{C} 11-\mathrm{C} 10-\mathrm{N} 1-\mathrm{C} 7$ & $-138.82(13)$ \\
\hline $\mathrm{C} 11-\mathrm{C} 10-\mathrm{N} 1-\mathrm{C} 13$ & $65.57(15)$ \\
\hline $\mathrm{O} 3-\mathrm{C} 17-\mathrm{N} 2-\mathrm{C} 18$ & $-10.2(2)$ \\
\hline $\mathrm{C} 16-\mathrm{C} 17-\mathrm{N} 2-\mathrm{C} 18$ & $166.87(15)$ \\
\hline $\mathrm{C} 21-\mathrm{C} 18-\mathrm{N} 2-\mathrm{C} 17$ & $73.7(2)$ \\
\hline $\mathrm{C} 20-\mathrm{C} 18-\mathrm{N} 2-\mathrm{C} 17$ & $-168.28(15)$ \\
\hline $\mathrm{C} 19-\mathrm{C} 18-\mathrm{N} 2-\mathrm{C} 17$ & $-49.4(2)$ \\
\hline $\mathrm{O} 2-\mathrm{C} 9-\mathrm{O} 1-\mathrm{C} 1$ & $-177.30(13)$ \\
\hline $\mathrm{C} 8-\mathrm{C} 9-\mathrm{O} 1-\mathrm{C} 1$ & 4.37 (19) \\
\hline $\mathrm{C} 2-\mathrm{C} 1-\mathrm{O} 1-\mathrm{C} 9$ & $178.43(13)$ \\
\hline $\mathrm{C} 6-\mathrm{C} 1-\mathrm{O} 1-\mathrm{C} 9$ & $0.6(2)$ \\
\hline $\mathrm{N} 2-\mathrm{C} 17-\mathrm{O} 3-\mathrm{C} 14$ & $175.06(13)$ \\
\hline $\mathrm{C} 16-\mathrm{C} 17-\mathrm{O} 3-\mathrm{C} 14$ & $-2.50(16)$ \\
\hline $\mathrm{C} 15-\mathrm{C} 14-\mathrm{O} 3-\mathrm{C} 17$ & $1.49(15)$ \\
\hline
\end{tabular}




$\begin{array}{llll}\mathrm{O} 3-\mathrm{C} 14-\mathrm{C} 15-\mathrm{C} 16 & 0.03(16) & \mathrm{C} 8-\mathrm{C} 14-\mathrm{O} 3-\mathrm{C} 17 & -179.57(12) \\ \mathrm{C} 8-\mathrm{C} 14-\mathrm{C} 15-\mathrm{C} 16 & -178.64(15) & \mathrm{O} 4-\mathrm{C} 22-\mathrm{O}-\mathrm{C} 23 & -2.8(2) \\ \mathrm{O} 3-\mathrm{C} 14-\mathrm{C} 15-\mathrm{C} 24 & -178.07(12) & \mathrm{C} 16-\mathrm{C} 22-\mathrm{O} 5-\mathrm{C} 23 & 174.87(12) \\ \mathrm{C} 8-\mathrm{C} 14-\mathrm{C} 15-\mathrm{C} 24 & 3.3(3) & \mathrm{O} 6-\mathrm{C} 24-\mathrm{O} 7-\mathrm{C} 25 & 9.0(2) \\ \mathrm{C} 14-\mathrm{C} 15-\mathrm{C} 16-\mathrm{C} 17 & -1.50(16) & \mathrm{C} 15-\mathrm{C} 24-\mathrm{O} 7-\mathrm{C} 25 & -169.88(12) \\ \mathrm{C} 24-\mathrm{C} 15-\mathrm{C} 16-\mathrm{C} 17 & 176.60(13) & \mathrm{O} 1 \mathrm{~S}-\mathrm{C} 2 \mathrm{~S}-\mathrm{O} 2 \mathrm{~S}-\mathrm{C} 3 \mathrm{~S} & 9.0(14) \\ \mathrm{C} 14-\mathrm{C} 15-\mathrm{C} 16-\mathrm{C} 22 & -167.79(15) & \mathrm{C} 1 \mathrm{~S}-\mathrm{C} 2 \mathrm{~S}-\mathrm{O} 2 \mathrm{~S}-\mathrm{C} 3 \mathrm{~S} & -175(2) \\ \mathrm{C} 24-\mathrm{C} 15-\mathrm{C} 16-\mathrm{C} 22 & 10.3(2) & \mathrm{C} 4 \mathrm{~S}-\mathrm{C} 3 \mathrm{~S}-\mathrm{O} 2 \mathrm{~S}-\mathrm{C} 2 \mathrm{~S} & -174.6(5) \\ \mathrm{C} 22-\mathrm{C} 16-\mathrm{C} 17-\mathrm{N} 2 & -6.8(2) & \mathrm{C} 13-\mathrm{C} 12-\mathrm{S} 1-\mathrm{C} 11 & -53.91(11) \\ \mathrm{C} 15-\mathrm{C} 16-\mathrm{C} 17-\mathrm{N} 2 & -174.76(15) & \mathrm{C} 10-\mathrm{C} 11-\mathrm{S} 1-\mathrm{C} 12 & 52.18(11) \\ \mathrm{C} 22-\mathrm{C} 16-\mathrm{C} 17-\mathrm{O} 3 & 170.45(13) & & \end{array}$

Hydrogen-bond geometry $\left(\AA,{ }^{\circ}\right)$

\begin{tabular}{lllll}
\hline$D-\mathrm{H} \cdots A$ & $D-\mathrm{H}$ & $\mathrm{H} \cdots A$ & $D \cdots A$ & $D-\mathrm{H} \cdots A$ \\
\hline $\mathrm{N} 2-\mathrm{H} 13 \cdots \mathrm{O} 4$ & 0.86 & 2.25 & $2.8255(17)$ & 125 \\
$\mathrm{C} 3-\mathrm{H} 2 \cdots \mathrm{O} 6{ }^{\mathrm{i}}$ & 0.93 & 2.41 & $3.325(2)$ & 167 \\
$\mathrm{C} 12-\mathrm{H} 9 \cdots \mathrm{O} 44^{\mathrm{ii}}$ & 0.97 & 2.44 & $3.1531(18)$ & 130 \\
$\mathrm{C} 12-\mathrm{H} 10 \cdots \mathrm{O} 1 S^{\mathrm{iii}}$ & 0.97 & 2.48 & $3.208(5)$ & 132 \\
$\mathrm{C} 19-\mathrm{H} 15 \cdots \mathrm{O} 3$ & 0.96 & 2.42 & $3.0090(19)$ & 119 \\
$\mathrm{C} 23-\mathrm{H} 23 \cdots \mathrm{S} 1^{\text {iv }}$ & 0.96 & 2.78 & $3.5639(17)$ & 139 \\
$\mathrm{C} 25-\mathrm{H} 26 \cdots \mathrm{S}^{v}$ & 0.96 & 2.83 & $3.7406(18)$ & 159
\end{tabular}

Symmetry codes: (i) $-x+2,-y,-z+1$; (ii) $-x+1,-y,-z$; (iii) $x, y, z-1$; (iv) $-x+1, y+1 / 2,-z+1 / 2$; (v) $x,-y-1 / 2, z+1 / 2$. 